\title{
Editorial
}

\section{Kompetenzen und Materialien für digitales Lernen und online-Kommunikation}

\author{
Doris Lewalter und Sabine Walper \\ Technische Universität München, München \\ Deutsches Jugendinstitut, München
}

Die Bedeutung von digitalen Medien für Kommunikation und Lehr-Lern-Prozesse ist seit geraumer Zeit ein zentraler Gegenstand der Bildungsforschung und bildungspolitischer Diskussionen (z. B. Fischer, Mandl \& Todorova, 2010; Gräsel, Mandl, Manhart \& Kruppa, 2000; Reinmann, Hartung \& Florian, 2014). Digitale Medien durchdringen zunehmend unseren Alltag und sind in den meisten Berufen unabdingbar. Schulische Bildung soll durch die Vermittlung digitaler Medienkompetenzen hierauf vorbereiten und kann ihrerseits von den Vorteilen digitaler Medien für Lehr-LernProzesse profitieren. Der DigitalPakt Schule von Bund und Ländern reagiert auf den Bedarf wie auch die Potenziale digitaler Bildung und soll dazu beitragen, die technischen Voraussetzungen für einen breiteren Medieneinsatz im Unterricht zu schaffen. Dass in dieser Hinsicht beträchtlicher Bedarf besteht, hat sich nicht zuletzt in der Corona-Pandemie gezeigt. Durch den zeitweisen Shutdown haben digitale Medien enorm an Bedeutung gewonnen und waren weit überwiegend auch für den Kontakt zur Schule und schulisches Lernen unabdingbar. Sie sind zu einem zentralen Mittel nicht nur der Informationsübermittlung und des sozialen Austauschs, sondern auch der Gestaltung von Lehr-Lern-Prozessen auf Dis$\operatorname{tanz}$ geworden (Eickelmann \& Gerick, 2020).

Aber nicht erst seit der Corona-Pandemie wird das Lehren und Lernen mit digitalen Medien intensiv diskutiert. So nennen beispielsweise Eickelmann und Gerick (2017) folgende vier Zielperspektiven schulischen Lernens mit digitalen Medien: „die Vermittlung von Fer- tigkeiten im Umgang mit digitalen Medien, die Nutzung digitaler Medien zur Verbesserung des fachlichen Lernens, die Entwicklung und Umsetzung neuer Formen des Unterrichtens mit digitalen Medien sowie die Förderung des Medienkompetenzerwerbs bzw. des Erwerbs ,digitaler' Kompetenzen als Querschnittskompetenz" (Eickelmann \& Gerick, 2020, S. 155). Damit das schulische Lernen mit digitalen Medien erfolgreich verlaufen kann, bedarf es somit zum einen spezifischer medienbezogener Kompetenzen sowohl aufseiten der Lehrenden als auch der Lernenden. Zum anderen bedarf es geeigneter digitaler Lehr-Lern-Materialien und Angebote, die den jeweiligen Rahmenbedingungen gerecht werden.

Das vorliegende Themenheft leistet einen Beitrag zum Verständnis dieser Einflussfaktoren und eröffnet mögliche Perspektiven für deren Weiterentwicklung. Dazu gehen im ersten Beitrag Senkbeil, Ihme und Schöber auf die digitalen Kompetenzen von Lehrenden ein, während sich die nächsten beiden Artikel den digitalen Materialien als Ausgangsbasis für Lehr-LernProzesse und deren Effekten widmen. So analysieren Moser, Degner und Lewalter im zweiten Beitrag die den Lehrenden zur Verfügung stehenden digitalen Schulbücher im MINTBereich aus einer lehr-lernpsychologischen Perspektive. Ninaus, Kiili, Wortha und Moeller untersuchen anschließend im dritten Beitrag unter anderem die motivationalen Effekte eines Lernspiels zum Bruchrechnen. Im letzten Beitrag von Festl weitet sich die Perspektive, indem die sozialen Medienkompetenzen der Lernenden untersucht werden. 
Bei der Rezeption der Beiträge ist zu beachten, dass alle Beiträge weit vor Auftreten der Corona-Pandemie entstanden sind und folglich keine Bezüge zur aktuellen Situation bei Erscheinen des Themenhefts liefern können. Gleichwohl gewinnen die von ihnen behandelten Fragen angesichts der Erfahrungen während der Corona-Pandemie besondere Bedeutung.

\section{$\mathrm{Zu}$ den Beiträgen im Einzelnen:}

Martin Senkbeil, Jan Marten Ihme und Christian Schöber beschäftigen sich in ihrem Beitrag „Schulische Medienkompetenzförderung in einer digitalen Welt: Über welche digitalen Kompetenzen verfügen angehende Lehrkräfte?" auf der Basis einer Sekundäranalyse des Nationalen Bildungspanels (NEPS) mit den medienbezogenen Kompetenzen der zentralen Akteure im schulischen Lehr-Lerngeschehen, nämlich den Lehrenden. Ihre digitalen Kompetenzen sind von zentraler Bedeutung für die Umsetzung der eingangs genannten Zielperspektiven schulischen Lernens mit digitalen Medien. Die Autoren gehen dabei der Frage nach, über welche digitalen Kompetenzen Studierende zu Beginn des Studiums und im fortgeschrittenen Studium verfügen. Dabei werden Studierende des Lehramts mit Studierenden anderer Fachrichtungen verglichen, um die Annahme einer Negativselektion von Lehramtsstudierenden im Sinne geringerer digitaler Kompetenzen im Vergleich zu anderen Studierendengruppen zu prüfen. Die Befunde deuten für beide Gruppen von Lehramtsstudierenden auf Kompetenzdefizite im Vergleich zu Studierenden anderer Fachrichtungen hin. Insbesondere Lehramtsstudierende ohne mathematisch-naturwissenschaftliches Unterrichtsfach verfügen im Sinne einer negativen Binnenselektion über vergleichsweise geringe digitale Kompetenzen. Darüber hinaus erreicht ein beachtlicher Anteil der Studienteilnehmerinnen und -teilnehmer nicht die normativ festgelegten Mindeststandards digitaler Kompetenzen.

Damit schulisches Lernen mit digitalen Medien erfolgreich verlaufen kann, bedarf es neben den digitalen Kompetenzen der Lehrenden auch geeigneter Lehrmaterialien. Stephanie Moser, Miriam Degner und Doris Lewalter unterziehen daher in ihrem Beitrag „Digitale Schulbücher im MINT-Bereich: Eine Analyse vor dem Hintergrund lehr-lernpsychologischer Theorien" digitale Schulbücher einer eingehenden Untersuchung. Damit wird ein in vielen Fächern nach wie vor zentrales Lehrmedium der täglichen Unterrichtspraxis am Beispiel des MINT-Bereichs in den Fokus der Analyse gerückt. Der Beitrag geht der Frage nach, inwieweit die Gestaltung der auf dem Markt befindlichen und zugelassenen digitalen Schulbücher den Lehrenden und Lernenden die Vorteile multimedialen Lernens bietet. Dazu wird auf der Basis einschlägiger (medien-) und instruktionspsychologischer Theorien eine strukturierende Inhaltsanalyse von sieben Schulbüchern durchgeführt. Die Befunde ergeben ein heterogenes Bild, zeigen aber deutlich auf, dass die lehrlernpsychologischen Potenziale digitaler Schulbücher noch nicht ausgeschöpft werden.

Eine weitere Ausformung digitaler LehrLernmedien in Form von digitalen Lernspielen nehmen Manuel Ninaus, Kristian Kiili, Silke Maria Wortha und Korbinian Moeller in den Blick. Dieser Beitrag zur „Motivationsregulation bei Verwendung eines Lernspiels zur Messung des Bruchverständnisses in der Schule - Eine latente Profilanalyse" geht einer weiteren Zielperspektive schulischen Lernens mit digitalen Medien (Eickelmann \& Gerick, 2017) nach und befasst sich mit der Nutzung digitaler Medien zur Verbesserung des fachlichen Lernens. Dabei wird zum einen untersucht, inwieweit sich grundlegende Effekte der Forschung zu numerischer Kognition mit einem digitalen Bruchrechnen-Lernspiel zur Messung des Verständnisses von Brüchen replizieren lassen. Zum anderen wird erkundet, inwieweit spezifische Motivationsregulationsstrategien bei der Benutzung des Lernspiels identifiziert werden können. Anhand der Daten lassen sich drei Gruppen von Schülerinnen und Schülern identifizieren, die sich hinsichtlich der Verwendung von selbst- und fremdbestimmten Motivationsregulationsstrategien sowie dem wahrgenommen positiven Affekt während des Spielens unterscheiden. Damit werden motivationale Möglichkeiten (digitaler) Lernspiele im Schulunterricht aufgezeigt. 
Abschließend beschäftigt sich der Beitrag von Ruth Festl zu „Soziale Medienkompetenz von Jugendlichen - Zur Rolle von Wissen, Motivation und Fähigkeiten für ein sozial kompetentes Handeln online" mit der vierten von Eickelmann und Gerick (2017) genannten Zielperspektive schulischen Lernens mit digitalen Medien, die sich auf die Förderung des Medienkompetenzerwerbs bzw. des Erwerbs „digitaler“ Kompetenzen als Querschnittskompetenz bezieht. Festl geht hier der Konzeptualisierung und empirischen Untersuchung der sozialen Dimension von Medienkompetenz im Jugendalter nach. Der Fokus der Studie liegt auf der Analyse verschiedener Kompetenzbereiche für ein sozial kompetentes Handeln im online-Kontext. Dabei geht es in der Studie von Festl weniger um das Medienwissen der Schülerinnen und Schüler als vielmehr um deren Fähigkeiten und Motivation und deren Bezug zu ihrem medienbezogenen Handeln. Festl befragt mit einem neu entwickelten standardisierten Messinstrument Schülerinnen und Schüler zu ihren Einschätzungen und Wahrnehmungen und ermittelt vier latente soziale Kompetenzfaktoren (partizipativ, moralisch, integrativ, vermittelnd), die sich jeweils aus Wissen, Motivation und Fähigkeiten zusammensetzen. Wie die Studie zeigt, wird das Handeln der Jugendlichen von allen vier Teilkomponenten, aber insbesondere von der Motivation beeinflusst. Festl zieht daraus den Schluss, dass die situativen Motive der Jugendlichen für ihr soziales online-Handeln bei der Förderung von sozialer Medienkompetenz in der pädagogischen Praxis stärker in den Blick genommen werden sollten.

\section{Literatur}

Eickelmann, B. \& Gerick, J. (2017). Lehren und Lernen mit digitalen Medien. Schulmanagement Handbuch, 164 (4), 54-81. http://dx.doi.org/10.31244/9783830 992318.09

Eickelmann, B. \& Gerick, J. (2020). Lernen mit digitalen Medien. Zielsetzungen in Zeiten von Corona und unter besonderer Berücksichtigung von sozialen Ungleichheiten. In D. Fickermann \& B. Edelstein (Hrsg.), „Langsam vermisse ich die Schule... ". Schule während und nach der Corona-Pandemie (S. 153-162). Münster, New York: Waxmann. http://dx.doi.org/10.31244/97 83830992318.09

Fischer F., Mandl H. \& Todorova A. (2010). Lehren und Lernen mit neuen Medien. In R. Tippelt \& B. Schmidt (Hrsg.), Handbuch Bildungsforschung (S. 753-771). Springer. https://doi.org/10.1007/978-3-531-920153_40

Gräsel, C., Mandl, H., Manhart, P. \& Kruppa, K. (2000). Das BLK-Programm" Systematische Einbeziehung von Medien, Informations-und Kommunikationstechnologien in Lehr-und Lernprozesse". Unterrichtswissenschaft, 28, 127-143.

Reinmann, G., Hartung, S. \& Florian, A. (2014). Akademische Medienkompetenz im Schnittfeld von Lehren, Lernen, Forschen und Verwalten. Grundbildung $\mathrm{Me}$ dien in pädagogischen Studiengängen, 319-332.

\section{Prof. Dr. Doris Lewalter}

Technische Universität München School of Education

Formelles und Informelles Lernen

Arcisstraße 21

D-80333 München

E-Mail: doris.lewalter@tum.de

\section{Prof. Dr. Sabine Walper}

DJI München

Deutsches Jugendinstitut e.V.

Nockherstr. 2

D-81541 München

E-Mail: walper@dji.de 